\title{
Lesões Músculo-Esqueléticas em Remadores de Competição
}

\author{
Musculoskeletal Injuries in Competitive Rowers
}

\author{
Francisco BERNARDES $\rrbracket^{1}$, Alfredo MENDES-CASTRO ${ }^{1,2}$, José RAMOS ${ }^{3}$, Ovídio COSTA $^{3}$ \\ Acta Med Port 2015 Jul-Aug;28(4):427-434
}

\section{RESUMO}

Introdução: O objetivo deste estudo foi a avaliação das lesões ocorridas ao longo da vida desportiva de remadores de competição seniores portugueses.

Material e Métodos: Enviámos um questionário a todos os remadores seniores medalhados nos campeonatos nacionais na época de 2013-2014. Analisámos variáveis biométricas, localização anatómica, tipo e circunstâncias de ocorrência de lesões, tratamentos efetuados e tempos de inatividade. Para análise estatística utilizámos métodos paramétricos e não paramétricos com níveis de confiança de $95 \%(p<0,05)$.

Resultados: Responderam 18 dos 18 remadores femininos e 74 dos 77 masculinos. Os femininos apresentaram idades, estaturas e pesos significativamente mais baixos e remaram mais parelhos $(p<0,05)$. O número de anos de prática e de lesões por remador foram semelhantes, entre femininos e masculinos. A idade média da primeira lesão foi significativamente mais precoce nos femininos $(p<0,001)$, nos quais se registaram mais lesões progressivas do que agudas, embora de forma não significativa. Tanto nos femininos como nos masculinos, a localização anatómica e o tipo de lesão mais frequentes foram a região lombar e a contratura muscular, com maior ocorrência no inverno e na primavera, em terra, durante os treinos. A inatividade por lesão foi superior nos femininos, embora de forma não significativa.

Discussão: Os resultados que diferiram dos reportados por outros autores podem estar relacionados com diferenças nas populações estudadas ou com outros fatores que necessitam de ser esclarecidos.

Conclusão: As lesões dos remadores de competição portugueses, associadas provavelmente a um nível competitivo menos expressivo dos nossos atletas, foram menos graves do que as geralmente referidas na literatura.

Palavras-chave: Lesões em Atletas; Portugal; Sistema Musculoesquelético/lesões.

\section{ABSTRACT}

Introduction: The objective of this study was the assessment of the injuries occurred over the competitive rowing lifetime of Portuguese senior rowers.

Material and Methods: We sent a questionnaire to all Portuguese senior rowers medalled in the national championships during the 2013-2014 season. We analysed biometric variables, anatomic location, type and circumstances of injury occurrence, type of treatment provided and time of inactivity. For statistical analysis we used parametric and non-parametric statistics with $95 \%$ confidence levels $(p$ $<0.05)$.

Results: The questionnaire was answered by 18 of the 18 female and 74 of the 77 male rowers. Females had significantly lower ages, heights and weights and rowed more often sculling boats $(p<0.05)$. The number of years of practice, as well as the number of injuries per rower was similar, for both females and males. The mean age at the first injury was significantly lower in females $(p<0.001)$, who also had more progressive lesions than acute ones, although not significantly. In both, the most frequent anatomical location and type of injury were the lumbar region and muscular pathology, with a higher occurrence in winter and spring, during land training. Females sustained longer periods of inactivity, but not significantly.

Discussion: The results that were different from those reported by other authors may be related with differences in the studied populations or with other factors that need to be clarified.

Conclusion: The injuries sustained by the Portuguese competitive rowers, probably associated with a lower competitive exposure of our athletes, were less severe than those generally reported in the literature.

Keywords: Athletic Injuries; Musculoskeletal System/injuries; Portugal.

\section{INTRODUÇÃO}

O remo mobiliza cerca de 1600 atletas federados em Portugal. ${ }^{1}$ Ao nível da alta competição, é uma prática desportiva exigente, ${ }^{2}$ tendo sido a terceira modalidade com maior número de participantes nos jogos olímpicos de $2008^{3}$ e de $2012 .{ }^{4}$ É um desporto considerado seguro, mas pode associar-se a lesões de instalação aguda ou progressiva, relacionadas com trauma agudo ou com sobrecarga física excessiva e prolongada. As lesões podem levar à interrupção da prática desportiva, ou mesmo ao seu abandono. ${ }^{2-5}$ Alguns autores reportam riscos de lesão superiores aos de alguns desportos de contacto, tais como o râguebi $e$ o boxe, nomeadamente durante o treino. ${ }^{6,7}$

Os estudos sobre o número de lesões acumuladas por atletas de remo ao longo da sua vida desportiva, sobre programas de prevenção e sobre as circunstâncias de ocorrência de lesões são escassos e têm incidido essencialmente

1. Mestrado Integrado em Medicina. Faculdade de Medicina. Universidade do Porto. Porto. Portugal.

2. Center for Health Technology and Services Research (CINTESIS). Porto. Portugal.

3. Medicina Desportiva. Departamento de Medicina. Faculdade de Medicina. Universidade do Porto. Porto. Portugal.

$\square$ Autor correspondente: Francisco Bernardes. xicoflbernardes@hotmail.com

Recebido: 05 de Março de 2015 - Aceite: 11 de Junho de 2015 | Copyright @ Ordem dos Médicos 2015 
sobre atletas de nível olímpico, ${ }^{2-4,6,7}$ com níveis competitivos superiores aos da generalidade dos atletas portugueses. É o caso do estudo retrospetivo de Hickey et al, que reporta as lesões registadas numa base de dados do Instituto Australiano de Desporto, ocorridas entre atletas de seleção nacional, femininos e masculinos, durante períodos de estágio com a duração média de 18,5 a 18,6 meses. ${ }^{6} \mathrm{O}$ mesmo aconteceu no estudo prospetivo de Wilson et al, que apresenta as lesões, comunicadas por telefone, ocorridas em 8 atletas Irlandeses de seleção femininos e 12 masculinos, ao longo de 12 meses. ${ }^{7}$ Por outro lado, no seu estudo retrospetivo, Teitz et al analisaram apenas a persistência de dor lombar em remadores de desporto escolar, 0 a 20 anos depois de concluída a sua atividade desportiva. ${ }^{8}$

O objetivo deste trabalho foi a avaliação das lesões músculo-esqueléticas ocorridas ao longo da vida desportiva de remadores de competição seniores portugueses que se mantêm em atividade competitiva. De acordo com pesquisa efetuada na Scielo e na PubMed com a query "(injuries or trauma or traumatic or lesion or lesions or pain or fracture or hernia) and (rowers or rowing)", é a primeira vez que tal estudo se realiza em Portugal.

\section{MATERIAL E MÉTODOS}

Enviámos um questionário a todos os remadores seniores, femininos e masculinos, medalhados nos campeonatos nacionais de fundo e de velocidade realizados em Portugal na época de 2013-2014, selecionados por consulta do portal da Federação Portuguesa de Remo, ${ }^{9}$ depois de um pedido de colaboração solicitado aos atletas, treinadores e

\section{Escalões competitivos}

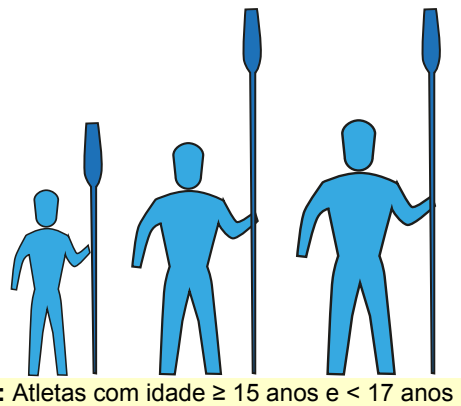

Juvenis: Atletas com idade $\geq 15$ anos e $<17$ anos Juniores: Atletas com idade $\geq 17$ anos e $<19$ anos Seniores: Atletas com idade $\geq 19$ anos responsáveis por clubes, levado a cabo durante o campeonato nacional de velocidade, em Julho de 2014.

Desenhámos o questionário com perguntas do tipo fechado, sobre género, idade, peso, estatura e categoria competitiva (pesado ou ligeiro) dos remadores, bem como sobre os barcos utilizados (parelhos ou pontas, isto é, com dois ou um remo por remador), a localização anatómica e o tipo de lesões sofridas, ao longo da vida desportiva do atleta, relacionadas com a prática da modalidade, em treino, ou em competição, no rio ou em terra. Colocámos ainda perguntas sobre as circunstâncias de ocorrência das lesões (escalão etário, mês, treino ou competição, tempo de treino e forma de instalação) e sobre o respetivo tratamento e consequências para a prática desportiva. Para descrição das lesões utilizaram-se expressões genéricas, próximas das adotadas em trabalhos semelhantes, facilmente compreensíveis pelos atletas. $6,7,10-12$

Definimos lesão como qualquer dor, incapacidade física ou outra situação semelhante que necessitou de tratamento, que tenha obrigado a faltar a algum treino ou prova. Incluímos esta definição nos questionários, nos quais apareceram também especificadas, sob a forma de pergunta fechada, as lesões mais frequentemente descritas, tais como, fratura, luxação, lesão muscular, tendinite, hérnia ou dor (quando para além desta queixa, nenhuma outra lesão foi evidente). Todos os tratamentos em que não houve referência a cirurgia, como repouso, fisioterapia ou fármacos foram considerados tratamento médico.

Aplicámos o questionário, previamente testado em dois atletas para clarificação das perguntas, via web e/ou em

\section{Categoria de peso}

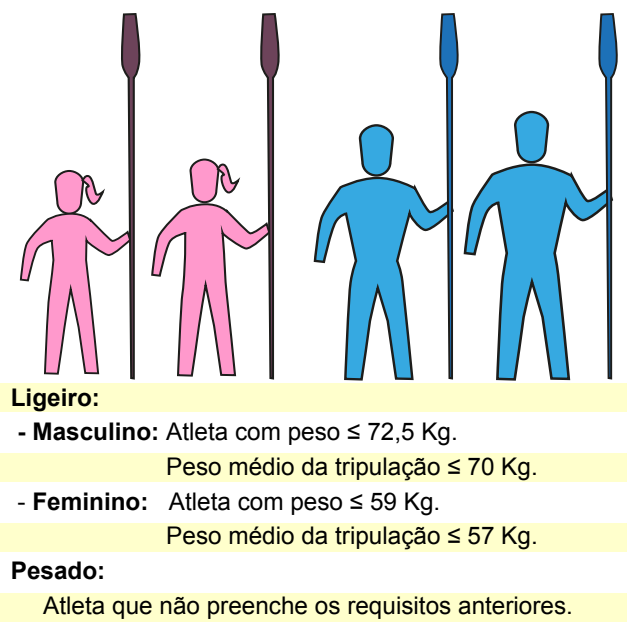

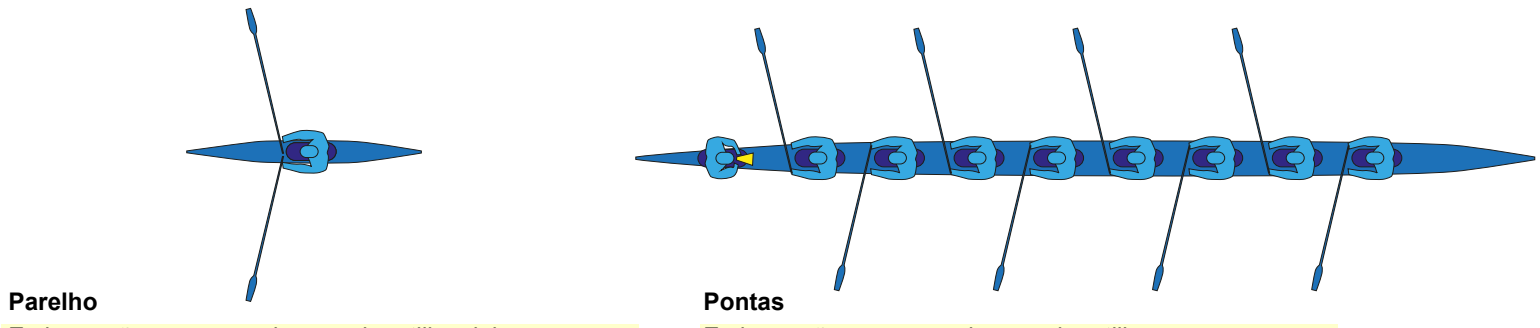

Embarcação em que cada remador utiliza dois remos

Embarcação em que cada remador utiliza um remo

Figura 1 - Definições técnicas relacionadas com a prática do remo 
presença física. A participação dos atletas foi sempre voluntária, depois de esclarecida a natureza e finalidade do estudo, bem como o tratamento anonimizado dos dados. As respostas foram sempre dadas de forma autónoma sem qualquer interferência por parte dos autores.

Os dados foram introduzidos numa base de dados anonimizada e foram validados pelos autores. Por uma questão de uniformização de critérios, expressões como 'fissura', 'rompimento' ou 'rotura' de ligamentos, foram consideradas numa mesma categoria, assim como 'fissura' ou 'fratura' óssea.

Estratificámos a análise de ocorrência de lesões, por género, em relação com o tempo dedicado a ações de prevenção, tipo de barco utilizado, localização anatómica, tipo de lesões sofridas, tipo de tratamento utilizado e impacto das lesões na atividade desportiva.

Efetuámos a análise estatística com o programa IBM SPSS Statistics (versão 22; Armonk, NY: IBM corp.) e Excel 2013 (2013; Redmond, WA: Microsoft Corp.). Avaliámos o tipo de distribuição das variáveis analisadas, com os testes de Kolmogorov-Smirnov e de Shapiro-Wilk. Apresentámos os resultados sob a forma de médias (desvio padrão), medianas (mínimo-máximo) ou proporções (intervalos de confiança de 95\% - IC 95\%), tendo utilizado para inferência estatística a análise dos IC 95\% e os testes t, Mann-Whitney ou Chi-quadrado, de acordo com o tipo de variável, com níveis de confiança de $95 \%(p<0,05)$. Para estimativa dos IC $95 \%$ utilizámos o método de Wilson sem correção para a continuidade.

O estudo foi autorizado pelo Comité de Ética do Departamento de Ciências Sociais e de Saúde da Faculdade de
Medicina da Universidade do Porto.

Na Fig. 1 apresentamos definições técnicas relacionadas com a prática do remo.

\section{RESULTADOS}

Obtivemos respostas de 92 dos 95 remadores elegíveis (18 de 18 femininos e 74 de 77 masculinos).

$\mathrm{Na}$ Tabela 1, verificamos que os remadores femininos apresentaram idades, estaturas e pesos significativamente mais baixos e remaram mais parelhos nos campeonatos nacionais do que os masculinos $(p<0,001)$. Contudo, a distribuição pelas categorias de pesados ou ligeiros, o número médio de anos de prática de remo, o número de sessões educativas de prevenção de lesões e os níveis de preocupação dos atletas com as mesmas, foram semelhantes entre femininos e masculinos. De salientar o baixo número de sessões educativas de prevenção de lesões, com valor mediano de zero sessões por ano.

A primeira lesão ocorreu significativamente mais cedo (17 versus 21 anos, $p<0,001$ ) nos atletas femininos do que nos masculinos, tendo, nestes últimos, surgido com maior significado estatístico no escalão sénior (83\%, IC 95\%: 75 - 88\%). Contudo, o número de lesões por atleta, ao longo das suas vidas desportivas de remadores de competição, bem como o risco global e de recidiva de lesão foram semelhantes entre femininos e masculinos (Tabela 2). Por outro lado, os atletas pesados, distribuídos de forma semeIhante por femininos e masculinos, apresentaram um risco de recidiva de lesão mais elevado do que os ligeiros $(90 \%$, IC 95\%: $77 \%$ - $96 \%$ versus $43 \%$, IC 95\%: 30 - 57\%, dados não apresentados em Tabela). Os oito atletas da seleção

Tabela 1 - Características biométricas e desportivas gerais dos 18 atletas femininos e 74 masculinos incluídos no estudo

\begin{tabular}{|c|c|c|c|c|c|c|c|}
\hline & \multicolumn{3}{|c|}{ Femininos } & \multicolumn{3}{|c|}{ Masculinos } & \multirow[t]{2}{*}{$p$} \\
\hline & $\mathbf{n}$ & & & $\mathbf{n}$ & & & \\
\hline Idade em anos mediana (min - máx) & 18 & 21 & $(19-43)$ & 72 & 24 & $(18-42)$ & 0,02 \\
\hline Estatura em centimetros - média $(d p)$ & 18 & 167 & $(1,4)$ & 73 & 181 & $(0,7)$ & $<0,001$ \\
\hline Peso em quilogramas - média (dp) & 18 & 60 & $(1,5)$ & 71 & 78 & (1) & $<0,001$ \\
\hline Categoria pesados - percentagem (IC 95\%) & 18 & 28 & $(13-51)$ & 74 & 45 & $(34-56)$ & \\
\hline Competição em seleção nacional - percentagem (IC 95\%) & 18 & 0 & $(0-18)$ & 74 & 11 & $(6-20)$ & \\
\hline Anos de vida desportiva média (dp) & 17 & 10 & $(1,2)$ & 70 & 12 & $(0,6)$ & 0,07 \\
\hline Número de treinos por semana mediana (min - máx) & 16 & 9 & $(5-14)$ & 71 & 7 & $(4-14)$ & 0,90 \\
\hline Número de horas por treino mediana (min - máx) & 16 & 2 & $(1,5-2)$ & 71 & 1,5 & $(1-2,5)$ & 0,20 \\
\hline Preocupação com lesões - percentagem (IC 95\%) & 17 & 88 & $(66-97)$ & 74 & 76 & $(65-84)$ & \\
\hline Sessões educativas na última época mediana (min - máx) & 8 & 0 & $(0-2)$ & 60 & 0 & $(0-5)$ & 0,60 \\
\hline \multicolumn{8}{|l|}{ Tipo de barco remado nos campeonatos nacionais } \\
\hline Parelhos - percentagem (IC 95\%) & 18 & 100 & $(82-100)$ & 74 & 20 & $(13-31)$ & \\
\hline Pontas - percentagem (IC 95\%) & 18 & 0 & $(0-18)$ & 74 & 49 & $(38-60)$ & \\
\hline Parelhos e pontas - percentagem (IC 95\%) & 18 & 0 & $(0-18)$ & 74 & 31 & $(22-42)$ & \\
\hline
\end{tabular}

n: Número de respostas dadas por cada variável; dp: Desvio padrão; IC 95\%: Intervalo de confiança de 95\%. 
Tabela 2 - Número, risco geral e risco de recidiva de lesões, por atleta, e idade, em anos, e escalão em que surgiu a primeira lesão, ao longo da vida dos 18 atletas femininos e 74 masculinos incluídos no estudo

\begin{tabular}{|c|c|c|c|c|c|c|c|}
\hline & \multicolumn{3}{|c|}{ Femininos } & \multicolumn{3}{|c|}{ Masculinos } & \multirow[t]{2}{*}{$p$} \\
\hline & $\mathbf{n}$ & & & $\mathbf{n}$ & & & \\
\hline Número de lesões/atleta mediana (min - máx) & 18 & 1 & $(0-6)$ & 74 & 1 & $(0-8)$ & 0,38 \\
\hline Risco de lesão/atleta proporção (IC 95\%) & 18 & 0,67 & $(0,44-0,84)$ & 74 & 0,80 & $(0,69-0,87)$ & \\
\hline Risco de recidiva de lesão/atleta proporção (IC 95\%) & 12 & 0,67 & $(0,39-0,86)$ & 59 & 0,61 & $(0,48-0,72)$ & \\
\hline Idade em que surgiu primeira lesão média (dp) & 9 & 17 & $(0,8)$ & 55 & 21 & $(0,6)$ & $<0,001$ \\
\hline \multicolumn{8}{|l|}{ Escalão em que surgiu primeira lesão } \\
\hline Juvenis-Juniores - percentagem (IC 95\%) & 20 & 35 & $(18-57)$ & 126 & 17 & $(12-25)$ & \\
\hline Seniores - percentagem (IC 95\%) & 20 & 65 & $(43-82)$ & 126 & 83 & $(75-88)$ & \\
\hline
\end{tabular}

nacional incluídos no estudo (todos masculinos e ligeiros; dois remadores de parelhos, quatro de pontas e dois de parelhos e pontas) apresentaram um risco global e de recidiva de lesão de 100\% (IC 95\%: 68 - 100\%) e de 33\% (IC 95\%: 1 - 70\%), respetivamente (dados não apresentados em Tabela).

Globalmente, a maior parte das lesões ocorreu durante os treinos, no inverno e na primavera (Tabela 3). Nos remadores femininos o principal tipo de atividade associado a lesões decorreu no ginásio e nos masculinos no rio. Os femininos apresentaram ainda significativamente mais lesões em competição do que os masculinos (24\%, IC 95\%: 11,5 - 43,4\% versus 4,2\%, IC 95\%: 1,8 - 9,4\%), nomeadamente a remar no rio $(20 \%$, IC 95\%: $8,9-39,1 \%$ versus $2,5 \%$, IC 95\%: 0,9 - 7,1\%). A maior parte das lesões ocorreu a remar parelhos entre os atletas femininos e a remar pontas entre os masculinos.

Os remadores femininos registaram mais lesões de instalação progressiva/sobrecarga (68,4\%, IC 95\%: 46,0 - $84,6 \%$ ) do que aguda (31,6\%, IC 95\%: 15,4 - 54,0\%), embora de forma não significativa, não se tendo verificado qualquer diferença entre os masculinos (Tabela 4). Por outro lado, a localização anatómica e o tipo de lesão mais frequentes, foram a região lombar $(33,3$ e $31,8 \%)$ e a contratura muscular $(29,2$ e $27,2 \%)$, tanto em femininos como masculinos.

A maioria dos tratamentos foi médico, tanto nos remadores femininos como masculinos, tendo-se registado, no entanto, uma tendência, sem significado estatístico, para durações mais longas de tratamento (21 versus 14 dias), de interrupção de treinos (14 versus 7 dias) e de treino condicionado, por lesão (30 versus 14 dias), entre os femininos (Tabela 5). Na Tabela 6, apresentamos uma análise descritiva dos casos sujeitos a tratamento cirúrgico.

\section{DISCUSSÃO}

Avaliámos as circunstâncias de ocorrência de lesões ao longo da vida desportiva de remadores de competição portugueses, baseados nas respostas dadas a um questionário, por não termos encontrado nenhum registo institu- cional, ou outro, com os dados pretendidos. Esta situação é semelhante à da maior parte dos estudos da mesma natureza, ${ }^{13,14}$ sendo raros os trabalhos que se basearam na análise de registos clínicos pré-existentes, ${ }^{3,4,6}$ bem como os estudos prospetivos. ${ }^{7}$ São conhecidas as limitações dos estudos retrospectivos, pelo que foi necessária uma validação cuidadosa dos dados, bem como uma análise cautelosa dos resultados.

A seleção da população estudada, constituída por todos os remadores medalhados em campeonatos nacionais numa época desportiva, foi pragmática tendo em vista uma representação dos nossos remadores mais qualificados. $O$ elevado número de remadores que participou no estudo ultrapassa o reportado por outros autores, tais como Wilson et al, que reportaram 20 participações entre 26 candidatos elegíveis. $^{7}$

Constatámos diferenças entre as caraterísticas dos remadores femininos e masculinos estudados (Tabela 1), que podem explicar algumas das diferenças evidenciadas nos padrões de lesão encontrados. As diferenças biométricas, tipicamente associadas ao género, afiguram-se particularmente importantes, havendo vários estudos, do âmbito da biomecânica, que apontam para uma maior suscetibilidade do biótipo feminino a certo tipo de lesões, nomeadamente do tórax. ${ }^{15-17}$ Já no que respeita às diferenças encontradas quanto aos tipos de barcos em que os atletas remaram, a situação não é clara. Só nos é possível alguma reflexão sobre os remadores masculinos, na medida em que estes remaram em vários tipos de barcos, ao contrário dos femininos, que remaram exclusivamente parelhos, sendo de referir que encontrámos relação estatisticamente significativa entre lesões e tipo de barco utilizado (Tabela 3). Wilson et al não encontraram diferenças estatisticamente significativas de lesões entre remadores de parelhos ou pontas. No entanto, esses autores encontraram lesões da coluna cervical exclusivamente em remadores de parelhos, ${ }^{7}$ o que não aconteceu no nosso estudo.

O número de sessões educativas, dedicadas à prevenção de lesões foi muito baixo. Mais de metade dos atletas respondeu zero sessões na última época (Tabela 1). Este 
Tabela 3 - Tipo de atividade, tipo de barco, carga de treino e mês de ocorrência associados às lesões registadas ao longo da vida dos 18 atletas femininos e 74 masculinos incluídos no estudo.

\begin{tabular}{|c|c|c|c|c|c|}
\hline & \multicolumn{2}{|c|}{ Femininos } & \multicolumn{2}{|c|}{ Masculinos } & $p$ \\
\hline \multicolumn{2}{|l|}{ Tipo de atividade - percentagem (IC 95\%) } & $\mathrm{n}=25$ & \multicolumn{2}{|c|}{$n=120$} & \\
\hline Treino & 76,0 & $(56,6-88,5)$ & 95,9 & $(90,8-98,2)$ & \\
\hline A remar no rio & 24,0 & $(11,5-43,4)$ & 40,2 & $(31,9-49,0)$ & \\
\hline No ergómetro & 8,0 & $(2,2-24,5)$ & 9,0 & $(5,1-15,4)$ & \\
\hline No ergómetro e a remar no rio & 12,0 & $(4,2-30)$ & 7,4 & $(3,9-13,4)$ & \\
\hline No ginásio & 28,0 & $(14,3-47,6)$ & 18,0 & $(12,2-25,8)$ & \\
\hline A correr & 4,0 & $(0,7-19,5)$ & 10,7 & $(6,3-17,4)$ & \\
\hline Outros * & 0,0 & $(0,0-13,3)$ & 10,7 & $(6,3-17,4)$ & \\
\hline Competição & 24,0 & $(11,5-43,4)$ & 4,1 & $(1,8-9,2)$ & \\
\hline A remar no rio & 20,0 & $(8,9-39,1)$ & 2,5 & $(0,8-7,0)$ & \\
\hline No ergómetro & 4,0 & $(0,7-19,5)$ & 1,6 & $(0,5-5,8)$ & \\
\hline Tipo de barco - percentagem (IC 95\%) & & $=20$ & & $=129$ & \\
\hline Parelhos & 90,0 & $(69,9-97,2)$ & 30,2 & $(22,3-38,6)$ & \\
\hline Pontas & 5,0 & $(0,9-23,6)$ & 59,7 & $(51,1-67,8)$ & \\
\hline Parelhos e pontas & 5,0 & $(0,9-23,6)$ & 10,1 & $(6,0-16,5)$ & \\
\hline \multicolumn{6}{|l|}{ Carga de treino } \\
\hline Treinos/semana n média $(\mathrm{dp})$ & 19 & $8,3(0,6)$ & 127 & $8,5(0,2)$ & 0,9 \\
\hline Horas/dia n mediana (min - máx) & 19 & $2(1,5-2)$ & 125 & $1,5(1-2,5)$ & 0,2 \\
\hline Mês de ocorrência - percentagem (IC 95\%) & \multicolumn{2}{|c|}{$\mathrm{n}=19$} & \multicolumn{2}{|c|}{$\mathrm{n}=98$} & \\
\hline Janeiro & 10,5 & $(2,9-31,4)$ & 16,3 & $(10,3-24,9)$ & \\
\hline Fevereiro & 5,3 & $(0,9-24,6)$ & 8,2 & $(4,2-15,3)$ & \\
\hline Março & 5,3 & $(0,9-24,6)$ & 12,2 & $(7,2-20,2)$ & \\
\hline Abril & 10,5 & $(2,9-31,4)$ & 6,1 & $(2,8-12,7)$ & \\
\hline Maio & 26,3 & $(11,8-48,8)$ & 13,3 & $(7,9-21,4)$ & \\
\hline Junho & 5,3 & $(0,9-24,6)$ & 6,1 & $(2,8-12,7)$ & \\
\hline Julho & 0,0 & $(0,0-16,8)$ & 7,1 & $(3,5-14,0)$ & \\
\hline Agosto & 0,0 & $(0,0-16,8)$ & 1 & $(0,2-5,6)$ & \\
\hline Setembro & 5,3 & $(0,9-24,6)$ & 9,2 & $(4,9-16,5)$ & \\
\hline Outubro & 5,3 & $(0,9-24,6)$ & 5,1 & $(2,2-11,4$ & \\
\hline Novembro & 10,5 & $(2,9-31,4)$ & 12,2 & $(7,2-20,2)$ & \\
\hline Dezembro & 15,8 & $(5,5-37,6)$ & 3,1 & $(1,1-8,6)$ & \\
\hline
\end{tabular}

\footnotetext{
n: Número de respostas dadas por cada variável; dp: Desvio padrão; IC 95\%: Intervalo de confiança de 95\%.
}

* em diversos tipos de combinação das atividades anteriores, a transportar o barco ou com bicicleta

aspeto, abordado por alguns autores, mas não especificamente em relação ao remo, merece mais investigação, dada a importância da prevenção de lesões na saúde e dinâmica desportiva dos atletas.

O número de 1,0 lesões, por atleta (feminino ou masculino), por vida desportiva média de 12 anos (Tabela 2), contrasta com os de Hickey et al, que apontam para um número médio de lesões muito superior, de 0,85 a 1,51 lesões por ano, entre atletas femininos e masculinos respeti- vamente ${ }^{6}$ bem como com os de Wilson et al, que apontam para uma média de 2,2 lesões por ano $(n=20) .{ }^{7}$ A maior ocorrência de lesões reportada por outros autores poderá ser explicada por caraterísticas individuais dos atletas por eles estudados, bem como por uma maior exposição ao esforço desses atletas, todos de nível olímpico.,7

Não encontrámos em nenhum estudo, para além do nosso, uma análise de risco de recidiva de lesão, que entre nós foi superior nos atletas pesados. Verificámos ainda que 
Tabela 4 - Forma de instalação, localização e tipo de lesões sofridas ao longo da vida dos 18 atletas femininos e 74 masculinos incluídos no estudo

\begin{tabular}{|c|c|c|c|c|}
\hline & \multicolumn{2}{|c|}{ Femininos } & \multicolumn{2}{|c|}{ Masculinos } \\
\hline Forma de instalação - percentagem (IC 95\%) & \multicolumn{2}{|c|}{$\mathrm{n}=19$} & \multicolumn{2}{|c|}{$\mathrm{n}=116$} \\
\hline Progressiva & 68,4 & $(46,0-84,6)$ & 50,0 & $(41,0-59,0)$ \\
\hline Aguda & 31,6 & $(15,4-54,0)$ & 50,0 & $(41,0-59,0)$ \\
\hline Localização - percentagem (IC 95\%) & \multicolumn{2}{|c|}{$\mathrm{n}=27$} & \multicolumn{2}{|c|}{$n=129$} \\
\hline Região cervical & 3,7 & $(0,7-18,3)$ & 5,4 & $(2,7-10,8)$ \\
\hline Região lombar & 33,3 & $(18,6-52,2)$ & 31,8 & $(24,4-40,3)$ \\
\hline Ombro & 25,9 & $(13,2-44,7)$ & 12,4 & $(7,8-19,2)$ \\
\hline Braço, antebraço e mão & 7,4 & $(2,1-23,4)$ & 15,2 & $(10,3-27,7)$ \\
\hline Costela & 0,0 & $(0,0-12,5)$ & 4,7 & $(2,2-9,8)$ \\
\hline Joelho & 18,5 & $(8,2-36,7)$ & 14,7 & $(9,6-21,9)$ \\
\hline Coxa, perna e pé & 11,1 & $(3,9-28,1)$ & 9,4 & $(5,4-15,6)$ \\
\hline Pelve & 0,0 & $(0,0-12,5)$ & 6,2 & $(3,2-11,8)$ \\
\hline Tipo - percentagem (IC 95\%) & \multicolumn{2}{|c|}{$\mathrm{n}=24$} & \multicolumn{2}{|c|}{$n=125$} \\
\hline Contratura muscular * & 29,2 & $(14,9-49,2)$ & 27,2 & $(20,2-35,6)$ \\
\hline Tendinite & 20,8 & $(8,2-40,5)$ & 25,6 & $(18,8-33,9)$ \\
\hline Dor & 20,8 & $(9,2-40,5)$ & 16,0 & $(10,6-23,4)$ \\
\hline Luxação/subluxação & 12,5 & $(4,3-31,0)$ & 7,2 & $(3,8-13,1)$ \\
\hline Hérnia & 12,5 & $(4,3-31,0)$ & 6,4 & $(3,3-12,1)$ \\
\hline Fissura/Fratura óssea & 4,2 & $(0,7-20,2)$ & 8,0 & $(4,4-14,1)$ \\
\hline Rotura muscular/tendinosa & 0,0 & $(0,0-13,8)$ & 2,4 & $(0,8-6,8)$ \\
\hline Outro ** & 0,0 & $(0,0-13,8)$ & 5,6 & $(2,7-11,1)$ \\
\hline
\end{tabular}

n: Número de respostas dadas por cada variável; dp: Desvio padrão; IC 95\%: Intervalo de confiança de 95\%.

* 1 feminino e 4 masculinos responderam fadiga muscular.

** 2 sacroileítes, 1 rotura de menisco, 1 sindroma de compartimento do antebraço, 1 apofisite.

Tabela 5 - Tipo de tratamento, por lesão, bem como dias de tratamento, de interrupção de atividades desportivas e de treino condicionado, por lesão, nos 18 atletas femininos e 74 masculinos incluídos no estudo.

\begin{tabular}{|c|c|c|c|c|c|}
\hline & \multicolumn{2}{|c|}{ Femininos } & \multicolumn{2}{|c|}{ Masculinos } & \multirow[t]{2}{*}{$p$} \\
\hline Tipo de tratamento/lesão & & $n=27$ & & $=126$ & \\
\hline Médico - percentagem (IC 95\%) & 96,3 & $(81,7-99,3)$ & 95,2 & $(90,0-97,8)$ & \\
\hline \multirow[t]{2}{*}{ Cirúrgico - percentagem (IC 95\%) } & 3,7 & $(0,6-18,2)$ & 4,8 & $(2,2-10,0)$ & \\
\hline & & $n=25$ & & $=119$ & \\
\hline Dias de tratamento/lesão n mediana (min - máx) & 21 & $(0-730)$ & 14 & $(0-540)$ & 0,45 \\
\hline Dias de interrupção de treino/lesão n mediana (min - máx) & 14 & $(0-730)$ & 7 & $(0-730)$ & 0,57 \\
\hline Dias de treino condicionado/lesão n mediana (min - máx) & 30 & $(0-730)$ & 14 & $(0-300)$ & 0,08 \\
\hline
\end{tabular}

a primeira lesão ocorreu significativamente mais cedo, e em escalões desportivos mais jovens, nos atletas femininos do que nos masculinos. Este aspeto, associado a uma idade significativamente mais jovem dos atletas femininos, com tempos de vida desportiva semelhantes aos masculinos, pode indiciar que os atletas femininos iniciam e terminam a prática de remo mais cedo, com padrões de risco de lesão diferentes. Estes dados devem suscitar a nossa 
Tabela 6 - Casos tratados com cirurgia, com indicação das principais características dos atletas e das lesões

\begin{tabular}{|c|c|c|c|c|c|c|c|c|c|}
\hline Caso & Género & Categoria & Escalão & Barco & $\begin{array}{c}\text { Tipo de } \\
\text { Atividade }\end{array}$ & Instalação & $\begin{array}{l}\text { Localização } \\
\text { Anatómica }\end{array}$ & Lesão & $\begin{array}{l}\text { Tempo de } \\
\text { Inatividade }\end{array}$ \\
\hline 1 & $M$ & Ligeiro & Júnior & Parelhos & Corrida & Aguda & Tornozelo & $\begin{array}{c}\text { Rotura } \\
\text { ligamentos }\end{array}$ & 90 \\
\hline 2 & $M$ & Ligeiro & Júnior & Pontas & Treino em rio & Progressiva & Joelho & $\begin{array}{l}\text { Rotura } \\
\text { menisco }\end{array}$ & 30 \\
\hline 3 & M & Ligeiro & Sénior & Parelhos & Ginásio & Progressiva & Joelho & $\begin{array}{l}\text { Fissura do } \\
\text { rotuliano }\end{array}$ & 450 \\
\hline 4 & $M$ & Ligeiro & Sénior & Pontas & $\begin{array}{l}\text { Ginásio e } \\
\text { ergómetro }\end{array}$ & Súbita & Região lombar & Hérnia L5-S1 & 90 \\
\hline 5 & $M$ & Pesado & Sénior & Pontas & Corrida & NR & Joelho & Fratura & 150 \\
\hline 6 & M & Pesado & Sénior & Pontas & Ginásio & Progressiva & Joelho & Tendinite & 7 \\
\hline 7 & $\mathrm{~F}$ & Ligeiro & Sénior & Parelhos & Ginásio & Progressiva & Joelho & Desvio rótula & 730 \\
\hline
\end{tabular}

M: masculino; F: feminino. Tempo de inatividade, em estimativa de dias, por conversão de respostas dadas em 'semanas', 'meses' ou 'anos'. NR: não respondeu.

atenção para um risco de lesão mais precoce entre atletas femininos, encontrado entre nós, bem como para um risco de lesão mais elevado entre atletas olímpicos, submetidos a cargas de esforço mais elevadas, encontrados por outros autores. ${ }^{6,7}$

A maior parte das lesões registadas ao longo da vida desportiva dos atletas ocorreu durante os treinos, no inverno e na primavera. Estes achados estão de acordo com a literatura que aponta para um maior risco de lesões em atividades de treino ${ }^{18}$ e durante os meses que antecedem as competições, que, em Portugal, ocorrem em março e juIho. ${ }^{9}$ De referir ainda a particularidade dos atletas femininos terem apresentado mais lesões em treino no ginásio e em competição no rio do que os masculinos. Este aspeto, que não foi avaliado em nenhuma outra publicação, sugere que os nossos atletas femininos podem apresentar-se a provas com menor preparação de treino em rio.

As lesões de instalação progressiva foram as mais frequentes entre remadores femininos, mas de forma não significativa. Essa forma de instalação também foi a mais frequente, mas de forma estatisticamente significativa, nos trabalhos de Yang $\mathrm{J}$ et $\mathrm{al},{ }^{5}$ Smoljanovic et $\mathrm{al}^{19} \mathrm{e}$ Hickey et $\mathrm{al}^{6}$ realizados, respetivamente, entre remadores de competição escolar, ou juniores e seniores de nível olímpico.

A localização anatómica e o tipo de lesão que encontrámos com maior frequência, tanto nos remadores femininos como masculinos, foram a região lombar e a contratura muscular. Estes resultados estão de acordo com Hickey et al no que respeita aos remadores masculinos, mas não em relação aos femininos, que apresentaram, nesse estudo, um predomínio de lesões torácicas, nomeadamente de fraturas de sobrecarga de costelas, ${ }^{6}$ muito em foco na literatura. ${ }^{20-24}$ Os nossos resultados estão também de acordo com Wilson et al no que respeita à localização mais frequente das lesões, mas em desacordo com estes autores, que registaram sobretudo lesões do tipo articular. ${ }^{7}$ Uma melhor caracterização anatómica, fisiopatológica e nosológica das lesões, a realizar em estudos futuros, poderá levar-nos a conclusões mais específicas. Este aspeto não foi possível, nem no nosso trabalho, nem nos estudos acima referidos,,${ }^{6,7}$ em que para a colheita de dados foi necessário elaborar questionários para serem respondidos por atletas sem conhecimentos médicos.

O tratamento da maior parte das lesões foi médico, tendo-se registado no entanto uma tendência, sem significado estatístico para durações mais longas, de tratamento e de inatividade por lesão entre os remadores femininos, em concordância com Hickey et al. ${ }^{6}$

O tratamento cirúrgico associou-se predominantemente a atletas masculinos e a lesões do joelho, embora de forma não estatisticamente significativa. Pelo contrário, Hickey et al encontraram mais casos cirúrgicos entre os atletas femininos, embora também sem significado estatístico. ${ }^{6}$

Para além das limitações já apontadas ao nosso estudo, e a outros, não deverá ser escamoteado que nenhum dos trabalhos realizados até hoje sobre lesões em remadores avaliou o número de atletas que deixou o remo por lesão, uma vez que todos os trabalhos incidiram apenas sobre remadores em atividade. Dessa forma, a incidência e a gravidade das lesões em remadores poderá estar a ser significativamente subvalorizada, deixando de fora os casos mais graves que tenham obrigado à interrupção permanente da prática desportiva.

Em estudos futuros deveremos tentar esclarecer se as diferenças encontradas entre o nosso estudo e os de outros autores se relacionaram apenas com a utilização de materiais e métodos diferentes ou também com outros tipos de fatores.

\section{CONCLUSÃO}

O nosso estudo mostrou pela primeira vez que as lesões dos remadores de competição portugueses são semelhantes às registadas na literatura, no que respeita à localização anatómica, tipo de lesão e circunstâncias de 
ocorrência. O estudo mostrou ainda, de novo, que os remadores femininos portugueses apresentam um risco de lesão em idade significativamente menor do que os masculinos e que os pesados apresentam um risco de recidiva de lesão mais elevado do que os ligeiros. O tempo dedicado pelos clubes a sessões de prevenção de lesões foi muito escasso e o tratamento cirúrgico associou-se predominantemente a atletas masculinos e a lesões do joelho. Estes dados, deverão ser tomados em consideração e justificam um esclarecimento mais aprofundado em estudos mais extensos.

\section{PROTECÇÃO DE PESSOAS E ANIMAIS / CONFIDEN- CIALIDADE DOS DADOS}

Os autores declaram que o estudo foi autorizado pelo Comité de Ética do Departamento de Ciências Sociais e de Saúde da Faculdade de Medicina da Universidade do Porto.

\section{REFERÊNCIAS}

1. Instituto Português do Desporto e Juventude; [consultado 2014 Set 9]. Disponível em: http://www.idesporto.pt/conteudo.aspx?id=103.

2. Shephard RJ. Science and medicine of rowing: a review. J Sports Sci. 1998;16:603-20.

3. Junge A, Engebretsen L, Mountjoy ML, Alonso JM, Renström PA, Aubry MJ, et al. Sports injuries during the Summer Olympic Games 2008. Am J Sports Med. 2009;37:2165-72.

4. Engebretsen L, Soligard T, Steffen K, Alonso JM, Aubry M, Budgett R, et al. Sports injuries and illnesses during the London Summer Olympic Games 2012. Br J Sports Med. 2013;47:407-14.

5. Yang J, Tibbetts AS, Covassin T, Cheng G, Nayar S, Heiden E. Epidemiology of overuse and acute injuries among competitive collegiate athletes. J Athl Train. 2012;47:198-204.

6. Hickey GJ, Fricker PA, McDonald WA. Injuries to elite rowers over a 10yr period. Med Sci Sports Exerc. 1997;29:1567-72.

7. Wilson F, Gissane C, Gormley J, Simms C. A 12-month prospective cohort study of injury in international rowers. $\mathrm{Br} \mathrm{J}$ Sports Med. 2010;44:207-14.

8. Teitz CC, O'Kane JW, Lind BK. Back pain in former intercollegiate rowers. A long-term follow-up study. Am J Sports Med. 2003;31:590-5

9. Federação Portuguesa de Remo; [consultado 2014 Set 9]. Disponível em: https://www.facebook.com/FederacaoPortuguesaRemo.

10. Hosea TM, Hannafin JA. Rowing injuries. Sports Health. 2012;4:236-45.

11. Karlson KA. Rowing injuries: identifying and treating musculoskeletal and nonmusculoskeletal conditions. Phys Sportsmed. 2000;28:40-50.

12. Karlson KA. Rowing: sport-specific concerns for the team physician. Curr Sports Med Rep. 2012;11:257-61.

13. Fernandes RS. Incidência, tipo e mecanismo de lesões no remo segundo a perceção dos remadores. Rev Bras Activ Fis Saude. 2007;12:181.

14. Antunes A, Duarte F, Santos-Carvalho M, Seara M, Oliveira P, Pinto R.

\section{AGRADECIMENTOS}

Ao Comité de Ética do Departamento de Ciências Sociais e de Saúde da Faculdade de Medicina da Universidade do Porto pelo parecer ético emitido. A Manuel Jorge Marques, estudante de doutoramento na Universidade de Kent, Reino Unido, pela ajuda na revisão do Abstract em Inglês. Ao presidente da Federação Portuguesa de Remo, Luís Ahrens Teixeira, pela colaboração na divulgação do estudo junto dos dirigentes dos clubes.

\section{CONFLITOS DE INTERESSES}

Os autores declaram a inexistência de conflitos de interesse.

\section{FONTES DE FINANCIAMENTO}

Não existiram fontes externas de financiamento para a realização deste artigo.
Influência da flexibilidade de isquiotibiais na lombalgia do remador estudo de 89 casos. Livro de resumos do XXXIII Congresso Nacional de Ortopedia e Traumatologia. Centro de Congressos do Algarve. 2013:33.

15. McNally E, Wilson D, Seiler S. Rowing injuries. Semin Musculoskelet Radiol. 2005:9:379-96.

16. Rumball JS, Lebrun CM, Di Ciacca SR, Orlando K. Rowing injuries Sports Med. 2005;35:537-55.

17. Howell DW. Muskuloskeletal profile and incidence of musculoskeletal injuries in lightweight women rowers. Am J Sports Med. 1984;12:278-82.

18. Brosh S, Jenner JR. Injuries to rowers. Br J Sports Med. 1988;22:169.

19. Smoljanovic T, Bojanic I, Hannafin JA, Hren D, Delimar D, Pecina M Traumatic and overuse injuries among international elite junior rowers. Am J Sports Med. 2009;37:1193-9.

20. McDonnell LK, Hume PA, Nolte V. Rib stress fractures among rowers definition, epidemiology, mechanisms, risk factors and effectiveness of injury prevention strategies. Sports Med. 2011;41:883-901.

21. Dragoni S, Giombini A, Di Cesare A, Ripani M, Magliani G. Stress fractures of the ribs in elite competitive rowers: a report of nine cases. Skeletal Radiol. 2007;36:951-4.

22. Vinther A, Kanstrup IL, Christiansen E, Alkjaer T, Larsson B, Magnusson $\mathrm{SP}$, et al. Exercise-induced rib stress fractures: potential risk factors related to thoracic muscle co-contraction and movement pattern. Scand J Med Sci Sports. 2006;16:188-96.

23. Vinther A, Kanstrup IL, Christiansen E, Alkjaer T, Larsson B, Magnusson $\mathrm{SP}$, et al. Exercise-induced rib stress fractures: influence of reduced bone mineral density. Scand J Med Sci Sports. 2005;15:95-9.

24. Smoljanovic T, Bojanic I, Pollock CL, Radonic R. Rib stress fracture in a male adaptive rower from the arms and shoulders sport class: case report. Croat Med J. 2011;52:644-7. 


\section{Lesões Músculo-Esqueléticas em Remadores de Competição}

Acta Med Port 2015:28:427-434

Publicado pela Acta Médica Portuguesa, a Revista Científica da Ordem dos Médicos

Av. Almirante Gago Coutinho, 151

1749-084 Lisboa, Portugal.

Tel: +351218428215

E-mail: submissao@actamedicaportuguesa.com

www.actamedicaportuguesa.com

ISSN:0870-399X | e-ISSN: 1646-0758

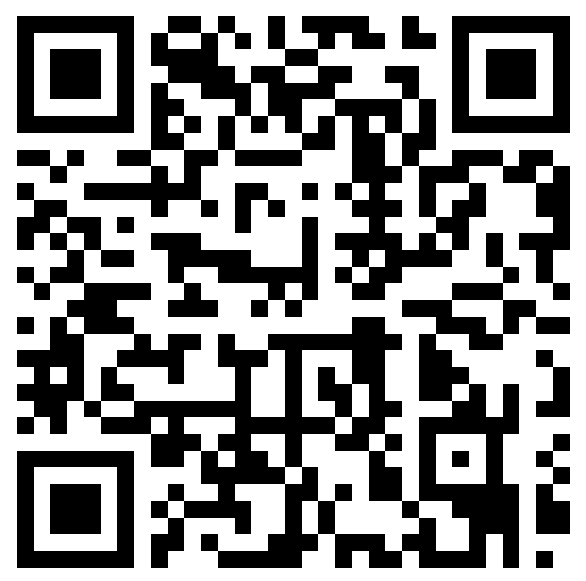

